\title{
Rethinking Outage Constraints for Resource Management in NOMA Networks
}

\author{
Fangyu Cui, Zhijin Qin, Yunlong Cai, Minjian Zhao, and Geoffrey Ye Li
}

\begin{abstract}
In non-orthogonal multiple access (NOMA) systems, the outage is regarded to happen when a user cannot correctly decode the messages for users with higher decoding order and hence cannot perform the successive interference cancellation (SIC). However, in this case, the user may still correctly decode its message by treating the uncancelled signal as interference and avoid the outage. By considering this behavior, the outage probability should be redefined. In this paper, we investigate user scheduling and power allocation for a downlink NOMA system with imperfect SIC by using the alternative outage probability as a constraint. In order to tackle the complicated non-convex resource allocation problem, we propose a two-phase algorithm, in which the user scheduling is first optimized through a matching theory based algorithm, and then power allocation is performed with the aid of the branch and bound $(B B)$ technique and the concave-convex procedure (CCCP) method. Simulation results show that the performance of the proposed low-complexity algorithm is near-optimal and the algorithm based on the alternative outage probability outperforms that based on the traditional one when the residual interference from imperfect SIC significantly affects the decoding.
\end{abstract}

Index Terms-NOMA, resource allocation, probabilistic constraints, imperfect SIC.

\section{INTRODUCTION}

Non-orthogonal multiple access (NOMA) has attracted much attention recently since it can effectively improve the capacity and support massive connectivity [1]-[6]. In traditional orthogonal multiple access (OMA), users are assigned to individual orthogonal resource blocks (RBs) to avoid the interference. While NOMA allows multiple user sharing the same RB with different power levels. At the receiver, successive interference cancellation (SIC) is employed to detect and decode the desired message. As a result, the number of supported users is increased with the cost of higher receiver complexity.

The basic concept of NOMA has been first proposed in [8]. Then, various aspects of the NOMA technique have been investigated. Usually, each user has a target rate determined by its required quality-of-service (QoS). An outage happens if the transmission rate is below the target rate. Hence, the outage probability becomes an important performance metric. In [9], stochastic geometry has been employed to analyze

F. Cui, Y. Cai and M. Zhao are with the College of Information Science and Electronic Engineering, Zhejiang University, Hangzhou 310027, China (e-mail: cfy531@zju.edu.cn; ylcai@zju.edu.cn; mjzhao@zju.edu.cn).

Z. Qin is with the School of Electronic Engineering and Computer Science, Queen Mary University of London, London E1 4NS, U.K. (email: z.qin@qmul.ac.uk).

G. Y. Li is with the School of Electrical and Computer Engineering, Georgia Institute of Technology, Atlanta, USA (e-mail: liye@ece.gatech.edu). the performance of NOMA systems by assuming that users are randomly distributed. Moreover, NOMA has been applied to other techniques, such as multiple-input multiple-output (MIMO) [10], cooperative communication [11], and heterogeneous network [12], for further performance improvement. A general framework for MIMO-NOMA has been proposed in [10], where the outage performance has been analyzed for both the uplink and downlink transmission. In [11], cooperative NOMA has been investigated as an approach of improving the outage performance by taking NOMA users closer to the base station as the relay. In [12], NOMA has been applied in a large-scale heterogeneous network to improve the spectrum efficiency. In [13], artificial noise (AN) has been applied to reduce the secrecy outage probability of NOMA systems. In [14], two effective schemes have been proposed to disrupt the potential eavesdropping in NOMA networks through joint precoding and jamming optimization.

In the aforementioned work on performance analyses, transmit power for each NOMA user is usually fixed. In order to further utilize the potential of NOMA, resources have been optimized to improve the system performance. Power allocation in NOMA systems has been investigated in [15] to maximize the sum rate. In [16], NOMA users have been carefully grouped to improve the throughput. In [17], the number of NOMA users in a cluster has been maximized by optimizing power allocation under the QoS and transmit power constraints. Furthermore, power allocation and user scheduling have been jointly optimized for NOMA systems [18][20]. In [21], channel assignment and power allocation have been optimized to maximize the energy efficiency of NOMA systems. In [22], user scheduling and power allocation have been optimized to improve the sum rate of a downlink millimeter wave (mmWave) NOMA system. Most of the works on resource allocation are based on the channel state information (CSI). However, in certain situation, average CSI is more realistic due to limited CSI feedback [23]. In [24], the outage balancing problem for NOMA systems with average CSI has been investigated. In [25], the power allocation has been considered to minimize the transmit power under the outage probability constraint. Moreover, in order to guarantee the user fairness, the transmission rate of the worst NOMA user has been maximized subject to the constraints of outage probability and transmit power.

The outage for a NOMA user can happen in the following cases according to the traditional outage probability definition [9], [25].

- The user cannot correctly decode the messages for the users with higher decoding order and hence cannot per- 
form the SIC successfully.

- The user cannot decode its own message even if it can decode the messages for users with higher decoding order.

However, even if a NOMA user cannot successfully perform the SIC, it may be still able to correctly decode the message by treating the uncancelled signal as interference. Therefore, the outage behavior has been redefined in [26] to provide a more accurate outage probability for the performance analysis of the NOMA system. As discussed in [26], the alternative outage probability is usually lower than the traditional one due to its more accurate definition. Hence, resource allocation based on the alternative outage probability, instead of the traditional one adopted in [25], should be able to achieve better performance and is worth investigating. Motivated by this, we investigate user scheduling and power allocation for a downlink NOMA network subject to the alternative outage probability constraints. Moreover, to make the problem more realistic, we consider imperfect SIC, i.e., there still exists residual interference after the cancellation process due to potential sources of errors such as channel estimation errors and decoding errors [26]-[30]. The main contributions are summarized as follows.

1) We formulate the user scheduling and power allocation problem for a downlink NOMA network to minimize transmit power subject to the alternative outage probability constraints under imperfect SIC assumption.

2) To handle the complicated problem, we decouple user scheduling and power allocation and propose a two-phase low-complexity suboptimal algorithm. The proposed user scheduling algorithm is based on matching theory [31]. The proposed power allocation algorithms are based on the branch and bound (BB) technique [32] and the concave-convex procedure (CCCP) method [33].

3 ) Based on our simulation results, the performance of the proposed matching theory based algorithm is close to that of the exhaustive search. Moreover, the total transmit power optimized based on the alternative outage probability is much lower than the traditional one, especially when the NOMA system is significantly affected by imperfect SIC.

The rest of the paper is organized as follows. In Section II, the system model is described and the problem is formulated. In Section III, a matching theory based user scheduling algorithm is proposed and its stability and complexity are analyzed. Section IV, power allocation algorithms are developed. The simulation results are shown in Section $\mathrm{V}$ and the conclusion is presented in Section VI.

\section{System Model AND Problem Formulation}

After introducing signal model and outage probability, we formulate the problem of user scheduling and power allocation.

\section{A. Signal Model}

We consider a downlink NOMA transmission scenario in a single cell, which consists one base station (BS) and $2 K$ users. The users are assumed uniformly distributed around the BS. Moreover, they are divided into $K$ orthogonal pairs and each pair is randomly assigned to a single RB. Without loss of generality, it is assumed that the distance ordering of the $2 K$ users obeys $r_{1} \leq \cdots \leq r_{n} \leq \cdots \leq r_{2 K}$.

If a user is assigned to $\mathrm{RB} k$ and its decoding order is $m$, we can denote its user index as $\pi_{k}(m)$. The signalplus-interference-to-noise ratio (SINR) of user $\pi_{k}(1)$ when decoding the message of itself can be expressed as

$$
\gamma_{k, 1}=\frac{\rho P_{\pi_{k}(1)} \lambda_{\pi_{k}(1)} h_{\pi_{k}(1)}}{\rho P_{\pi_{k}(2)} \lambda_{\pi_{k}(1)} h_{\pi_{k}(1)}+1},
$$

where $\sigma^{2}$ is the power of additive white Gaussian noise (AWGN) and $\rho=\frac{1}{\sigma^{2}}, P_{\pi_{k}(m)}$ is the power for user $\pi_{k}(m), h_{\pi_{k}(m)} \sim \exp (1)$ and $\lambda_{\pi_{k}(m)}$ refer to small-scale fading coefficient and large-scale fading coefficient between the BS and user $\pi_{k}(m)$, respectively. The large-scale fading coefficient is assumed as $\lambda_{\pi_{k}(m)}=\eta r_{\pi_{k}(m)}^{-\alpha}$, where $\eta$ is a frequency dependent factor, $r_{\pi_{k}(m)}$ is the distance between the BS and user $\pi_{k}(m)$, and $\alpha$ is the path loss exponent.

As for user $\pi_{k}(2)$, it will first decode the message of user $\pi_{k}(1)$ with the following SINR,

$$
\gamma_{k, 2 \rightarrow 1}=\frac{\rho P_{\pi_{k}(1)} \lambda_{\pi_{k}(2)} h_{\pi_{k}(2)}}{\rho P_{\pi_{k}(2)} \lambda_{\pi_{k}(2)} h_{\pi_{k}(2)}+1} .
$$

Then with SIC, user $\pi_{k}(2)$ cancels the interference from user $\pi_{k}(1)$. If the SIC process is successful, the SINR of user $\pi_{k}(2)$, when decoding its message, can be expressed as

$$
\gamma_{k, 2}=\frac{\rho P_{\pi_{k}(2)} \lambda_{\pi_{k}(2)} h_{\pi_{k}(2)}}{\rho P_{\pi_{k}(1)} \lambda_{\pi_{k}(2)} h_{\omega}+1} .
$$

Here we model the residual interference as $e_{\omega} \sim \mathcal{C N}(0, \omega)$, where $\omega$ denotes the variance of error [27]. Hence, the power of the residual interference follows the exponential distribution, i.e., $h_{\omega} \triangleq\left|e_{\omega}\right|^{2} \sim \exp \left(\frac{1}{\omega}\right)$. If the SIC process fails, user $\pi_{k}(2)$ can decode the message of itself by directly treating the signal for user $\pi_{k}(1)$ as interference. As such, the SINR can be expressed as

$$
\gamma_{k, 2 \rightarrow 2}=\frac{\rho P_{\pi_{k}(2)} \lambda_{\pi_{k}(2)} h_{\pi_{k}(2)}}{\rho P_{\pi_{k}(1)} \lambda_{\pi_{k}(2)} h_{\pi_{k}(2)}+1} .
$$

\section{B. Outage Probability}

The outage occurs when the receiving SINR of the user is below the required target SINR. Based on the above expression of SINR, the outage probability of user $\pi_{k}(1)$ can be expressed as

$$
\begin{aligned}
P_{\pi_{k}(1)}^{\text {out }} & =\operatorname{Pr}\left\{\gamma_{k, 1} \leq \phi_{\pi_{k}(1)}\right\} \\
& =\operatorname{Pr}\left\{h_{\pi_{k}(1)} \leq \frac{\phi_{\pi_{k}(1)}}{\rho \lambda_{\pi_{k}(1)}\left(P_{\pi_{k}(1)}-\phi_{\pi_{k}(1)} P_{\pi_{k}(2)}\right)}\right\} \\
& =1-e^{-\frac{\phi_{\pi_{k}(1)}}{\rho \lambda_{\pi_{k}(1)}\left(P_{\pi_{k}(1)}-\phi_{\pi_{k}(1)} P_{\pi_{k}(2)}\right)}}
\end{aligned}
$$

where $\phi_{\pi_{k}(1)}=2^{R_{\pi_{k}(1)}}-1$ is the target SINR, and $R_{\pi_{k}(1)}$ denotes the target rate of user $\pi_{k}(1)$.

As for the outage probability of user $\pi_{k}(2)$, there are two definitions as described below: 
1) Traditional Outage Probability: Consider a pair of users assigned to the RB $k$. In the traditional definition, the outage for user $\pi_{k}(2)$ can happen in the following two cases [9].

- User $\pi_{k}(2)$ cannot decode the message of user $\pi_{k}(1)$.

- User $\pi_{k}(2)$ can decode the message of user $\pi_{k}(1)$ and perform the SIC successfully, but cannot decode its own message.

From the outage behaviors analyzed above, the traditional outage probability of user $\pi_{k}(2)$ can be expressed as in (6), where $\phi_{\pi_{k}(2)}=2^{R_{\pi_{k}(2)}}-1$, and $g_{k}\left(P_{\pi_{k}(1)}, P_{\pi_{k}(2)}\right)$ is defined in (7).

2) Alternative Outage Probability: In the first outage case of the traditional definition, the outage will not happen if user $\pi_{k}(2)$ directly decode its own message successfully by treating the signal from user $\pi_{k}(1)$ as interference. In that case, the outage for user $\pi_{k}(2)$ can happen in the following two cases [26].

- User $\pi_{k}(2)$ cannot decode the message of user $\pi_{k}(1)$ and cannot decode its own message by treating the signal of user $\pi_{k}(1)$ as interference.

- User $\pi_{k}(2)$ can decode the message of user $\pi_{k}(1)$ and perform the SIC successfully, but cannot decode the message of itself.

By rethinking the outage behavior, the alternative outage probability for user $\pi_{k}(2)$ can be expressed as in (8).

It is worth noting that the alternative outage probability is different from the traditional one only when $\frac{\phi_{\pi_{k}(2)}}{P_{\pi_{k}(2)}-\phi_{\pi_{k}(2)} P_{\pi_{k}(1)}}<\frac{\phi_{\pi_{k}(1)}}{\text { If }_{\text {is }} \text { is }}$ perfect, the optimal order leads to $\frac{\phi_{\pi_{k}(2)}}{P_{\pi_{k}(2)}-\phi_{\pi_{k}(2)} P_{\pi_{k}(1)}} \geq \frac{\phi_{\pi_{k}(1)}}{P_{\pi_{k}(1)}-\phi_{\pi_{k}(1)} P_{\pi_{k}(2)}} \quad$ [25], then the two outage probability definitions become the same. As a result, the alternative outage probability may outperform the traditional one when the SIC is imperfect.

\section{Problem Formulation}

This paper aims to minimize the total transmit power subject to the outage probability constraints for each user by optimizing user scheduling and power allocation. The problem can be formulated as

$$
\begin{aligned}
\min _{\left\{P_{\left.\pi_{k}(m), \pi_{k}(m)\right\}}\right.} & \sum_{\forall k, m} P_{\pi_{k}(m)} \\
\text { s.t. } & P_{\pi_{k}(m)}^{\text {out }} \leq \epsilon_{\pi_{k}(m)}, \\
& P_{\pi_{k}(m)} \geq 0, \\
& \pi \in \Pi, \forall k, m,
\end{aligned}
$$

where $\epsilon_{\pi_{k}(m)}$ denotes the maximum allowed outage probability for user $\pi_{k}(m)$, and $\Pi$ denotes the set of all possible user scheduling, including the RB allocation and the decoding order in each RB.

The entanglement of user scheduling and power allocation makes the mixed-integer non-convex problem complicated. Hence, we decouple them and propose a two-phase algorithm. In the first phase, the user scheduling is optimized with given power allocation. In the second phase, the power allocation is optimized with given user scheduling obtained in the first phase $^{1}$. The more details are introduced in the following two sections.

\section{MATChing Theory BASED User Scheduling ALGORITHM}

In this section, we propose a low-complexity matching theory based user scheduling (MTUS) algorithm to avoid the high complexity of exhaustive search [31], [34], [35].

For given power allocation, the user scheduling problem can be formulated as

$$
\begin{aligned}
\min _{\pi_{k}(m)} & \sum_{\forall k, m} P_{\pi_{k}(m)} \\
\text { s.t. } & (9 \mathrm{~b})-(9 \mathrm{~d}) .
\end{aligned}
$$

With fixed power allocation, the objective function will not vary with different user scheduling schemes. However, user scheduling affects the outage probability for the system. (P1) aims to search for the user scheduling scheme that makes the outage probability of each user low enough to satisfy the QoS constraints with fixed power allocation. Therefore, we heuristically minimize the system outage probability, i.e., maximize the system coverage probability and formulate the following problem instead of $(\mathbf{P 1})$ :

$$
\begin{aligned}
\text { (P2) } \max _{\pi_{k}(m)} & \prod_{\forall k, m}\left(1-P_{\pi_{k}(m)}^{\text {out }}\right) \\
\text { s.t. } & \pi \in \Pi .
\end{aligned}
$$

Note that $(\mathbf{P 2})$ is not completely equivalent to $(\mathbf{P 1})$. However, they intuitively have the same purpose, i.e., reducing the outage probability by optimizing the user scheduling with fixed power. Moreover, since we aim to find a suboptimal solution with low complexity, $(\mathbf{P} 2)$ is adopted in the optimization of user scheduling. Moreover, the user to be decoded first is not necessarily allocated more power than the second user. Therefore, we only assume the fixed power allocated to the two pairing users in the $k$-th RB as $P_{k, a}>P_{k, b}$. The allocation of the fixed powers $P_{k, a}$ and $P_{k, b}$ to the pairing users $\pi_{k}(1)$ and $\pi_{k}(2)$ should be searched in the matching theory based algorithm proposed later.

\section{A. Preliminaries of Many-to-One Matching}

We first define the many-to-one matching model in this subsection. The sets of users and RBs are denoted as $\mathcal{N}$ and $\mathcal{K}$, respectively. As a result, the user scheduling problem can be regarded as matching the users in $\mathcal{N}$ with the RBs in $\mathcal{K}$. Hence, we can define a many-to-one matching $\boldsymbol{\Phi}$ as in [34]. If user $n \in \mathcal{N}$ and RB $k \in \mathcal{K}$ are matched, we have $n \in \mathbf{\Phi}(k)$ and $\boldsymbol{\Phi}(n)=k$.

In order to model the decision process of the matching, we define the preference relations $\succ_{n}$ over $\mathcal{K}$ and $\succ_{k}$ over $\mathcal{N}$ for user $n$ and RB $k$, respectively. The preference value for user $n$ under the matching $\boldsymbol{\Phi}$ is defined as the coverage probability

$$
U_{n}(\mathbf{\Phi})=1-P_{n}^{\text {out }},
$$

\footnotetext{
${ }^{1}$ There is no iteration between two phases since we aim at a low-complexity suboptimal algorithm instead of obtaining global optimal solution.
} 


$$
\begin{aligned}
& P_{\pi_{k}(2)}^{\text {out }, T R}=\operatorname{Pr}\left\{\gamma_{k, 2 \rightarrow 1} \leq \phi_{\pi_{k}(1)}\right\}+\operatorname{Pr}\left\{\gamma_{k, 2 \rightarrow 1}>\phi_{\pi_{k}(1)}, \gamma_{k, 2} \leq \phi_{\pi_{k}(2)}\right\} \\
& =\operatorname{Pr}\left\{h_{\pi_{k}(1)} \leq \frac{\phi_{\pi_{k}(1)}}{\rho \lambda_{\pi_{k}(2)}\left(P_{\pi_{k}(1)}-\phi_{\pi_{k}(1)} P_{\pi_{k}(2)}\right)}\right\} \\
& +\operatorname{Pr}\left\{\frac{\phi_{\pi_{k}(1)}}{\rho \lambda_{\pi_{k}(2)}\left(P_{\pi_{k}(1)}-\phi_{\pi_{k}(1)} P_{\pi_{k}(2)}\right)}<h_{\pi_{k}(2)} \leq \frac{\phi_{\pi_{k}(2)}\left(\rho \lambda_{\pi_{k}(2)} P_{\pi_{k}(1)} h_{\omega}+1\right)}{\rho \lambda_{\pi_{k}(2)} P_{\pi_{k}(2)}}\right\} \\
& =1-e^{-\frac{\phi_{\pi_{k}(1)}}{\rho \lambda_{\pi_{k}(2)}\left(P_{\pi_{k}(1)}-\phi_{\pi_{k}(1)} P_{\pi_{k}(2)}\right)}}+g_{k}\left(P_{\pi_{k}(1)}, P_{\pi_{k}(2)}\right) \text {. } \\
& g_{k}\left(P_{\pi_{k}(1)}, P_{\pi_{k}(2)}\right)=\max \left(e^{-\frac{\phi_{\pi_{k}(1)}}{\rho \lambda_{\pi_{k}(2)}\left(P_{\pi_{k}(1)}-\phi_{\pi_{k}(1)} P_{\pi_{k}(2)}\right)}}-\frac{P_{\pi_{k}(2)}}{P_{\pi_{k}(2)}+P_{\pi_{k}(1)} \omega \phi_{\pi_{k}(2)}} e^{-\frac{\phi_{\pi_{k}(2)}}{\rho \lambda_{\pi_{k}}(2) P_{\pi_{k}(2)}}}, 0\right) \\
& P_{\pi_{k}(2)}^{\text {out }, A L}=\operatorname{Pr}\left\{\gamma_{k, 2 \rightarrow 1} \leq \phi_{\pi_{k}(1)}, \gamma_{k, 2 \rightarrow 2} \leq \phi_{\pi_{k}(2)}\right\}+\operatorname{Pr}\left\{\gamma_{k, 2 \rightarrow 1}>\phi_{\pi_{k}(1)}, \gamma_{k, 2} \leq \phi_{\pi_{k}(2)}\right\} \\
& =\operatorname{Pr}\left\{h_{\pi_{k}(1)} \leq \min \left(\frac{\phi_{\pi_{k}(1)}}{\rho \lambda_{\pi_{k}(2)}\left(P_{\pi_{k}(1)}-\phi_{\pi_{k}(1)} P_{\pi_{k}(2)}\right)}, \frac{\phi_{\pi_{k}(2)}}{\rho \lambda_{\pi_{k}(2)}\left(P_{\pi_{k}(2)}-\phi_{\pi_{k}(2)} P_{\pi_{k}(1)}\right)}\right)\right\} \\
& +\operatorname{Pr}\left\{\frac{\phi_{\pi_{k}(1)}}{\rho \lambda_{\pi_{k}(2)}\left(P_{\pi_{k}(1)}-\phi_{\pi_{k}(1)} P_{\pi_{k}(2)}\right)}<h_{\pi_{k}(2)} \leq \frac{\phi_{\pi_{k}(2)}\left(\rho \lambda_{\pi_{k}(2)} P_{\pi_{k}(1)} h_{\omega}+1\right)}{\rho \lambda_{\pi_{k}(2)} P_{\pi_{k}(2)}}\right\} \\
& =1-e^{-\min \left(\frac{\phi_{\pi_{k}(1)}}{\rho \lambda_{\pi_{k}(2)}\left(P_{\pi_{k}(1)}-\phi_{\pi_{k}(1)} P_{\pi_{k}(2)}\right)}, \frac{\phi_{\pi_{k}(2)}}{\rho \lambda_{\pi_{k}(2)}\left(P_{\pi_{k}(2)}-\phi_{\pi_{k}(2)} P_{\pi_{k}(1)}\right)}\right)}+g_{k}\left(P_{\pi_{k}(1)}, P_{\pi_{k}(2)}\right) \text {. }
\end{aligned}
$$

where $P_{n}^{\text {out }}$ denotes the outage probability for user $n$ under the matching $\boldsymbol{\Phi}$. The relation that user $n$ prefers to match with RB $k=\boldsymbol{\Phi}(n)$ instead of $k^{\prime}=\boldsymbol{\Phi}^{\prime}(n)$ can be expressed as

$$
(k, \boldsymbol{\Phi}) \succ_{n}\left(k^{\prime}, \boldsymbol{\Phi}^{\prime}\right) \Leftrightarrow U_{n}(\boldsymbol{\Phi})>U_{n}\left(\boldsymbol{\Phi}^{\prime}\right) .
$$

Similarly, the preference value for RB $k$ is defined as

$$
U_{k}(\boldsymbol{\Phi})=\prod_{n \in \mathbf{\Phi}(k)}\left(1-P_{n}^{\text {out }}\right) .
$$

The relation that $\mathrm{RB} k$ prefers to match with the user set $\mathcal{C}=$ $\boldsymbol{\Phi}(k)$ instead of $\mathcal{C}^{\prime}=\boldsymbol{\Phi}^{\prime}(k)$ can be expressed as

$$
(C, \boldsymbol{\Phi}) \succ_{k}\left(C^{\prime}, \boldsymbol{\Phi}^{\prime}\right) \Leftrightarrow U_{k}(\boldsymbol{\Phi})>U_{k}\left(\boldsymbol{\Phi}^{\prime}\right) .
$$

Due to the interference between pairing users, changing the matching of one user will also affect the preference values of other users and hence the stability of the matching cannot be defined straightforwardly. To tackle this problem, two-sided exchange stability in [35] is employed in the design of the matching theory based algorithm. To better describe the twosided exchange stability, we first define swap matching as follows.

Definition 1. Given the matching $\boldsymbol{\Phi}$ with $k=\boldsymbol{\Phi}(n)$ and $k^{\prime}=\boldsymbol{\Phi}\left(n^{\prime}\right)$, a swap matching for $\boldsymbol{\Phi}$ is defined as $\boldsymbol{\Phi}^{\prime}=$ $\left\{\boldsymbol{\Phi} \backslash\left\{(k, n),\left(k^{\prime}, n^{\prime}\right)\right\} \cup\left\{\left(k, n^{\prime}\right),\left(k^{\prime}, n\right)\right\}\right\}$, where $k=\boldsymbol{\Phi}\left(n^{\prime}\right)$ and $k^{\prime}=\boldsymbol{\Phi}(n)$.

Note that the preference values cannot be directly updated after a swap operation between two users since the decoding order and the allocation of the fixed power coefficients are not determined. When user $n$ is swapped to match with a new $\mathrm{RB} k^{\prime}$, there are four possible cases for the allocation of decoding order and power coefficients for user $n$, which are listed as below.

1) User $n$ is allocated with power $P_{k, a}$ and the first to be decoded;

2) User $n$ is allocated with power $P_{k, a}$ and the second to be decoded;

3) User $n$ is allocated with power $P_{k, b}$ and the first to be decoded;

4) User $n$ is allocated with power $P_{k, b}$ and the second to be decoded.

We denote the preference values for user $n$ and $\mathrm{RB} k^{\prime}$ as $U_{n}^{i}$ and $U_{k^{\prime}}^{i}$, respectively, in the $i$-th case. Here, $i \in\{1,2,3,4\}$. Then we have the following definition.

Definition 2. Given a pair of users $\left(n, n^{\prime}\right)$ and a matching $\mathbf{\Phi}$, where $k=\boldsymbol{\Phi}(n)$ and $k^{\prime}=\boldsymbol{\Phi}\left(n^{\prime}\right)$, if there exist the cases $i, j \in$ $\{1,2,3,4\}$ such that

1) $\forall x \in\left\{n, k^{\prime}\right\}, U_{x}^{i}\left(\boldsymbol{\Phi}^{\prime}\right) \geq U_{x}(\boldsymbol{\Phi})$, and $\forall y \in$ $\left\{n^{\prime}, k\right\}, U_{y}^{j}\left(\boldsymbol{\Phi}^{\prime}\right) \geq U_{y}(\boldsymbol{\Phi})$;

2) $\exists x \in\left\{n, k^{\prime}\right\}$ such that $U_{x}^{i}\left(\mathbf{\Phi}^{\prime}\right)>U_{x}(\boldsymbol{\Phi})$, or $\exists y \in$ $\left\{n^{\prime}, k\right\}$ such that $U_{y}^{j}\left(\boldsymbol{\Phi}^{\prime}\right)>U_{y}(\mathbf{\Phi})$,

then the swap matching $\boldsymbol{\Phi}^{\prime}$ is approved, and $\left(n, n^{\prime}\right)$ is defined as a swap-blocking pair in $\mathbf{\Phi}$.

Definition 2 states that if the swap matching is approved, the preference values for all users will not decrease and the preference value of at least one user will increase. Moreover, the decoding order and power coefficients will be allocated according to the case that approves the swap matching. If a swap-blocking pair exists in the current matching $\boldsymbol{\Phi}$, we can swap them to improve system performance. After a number of 


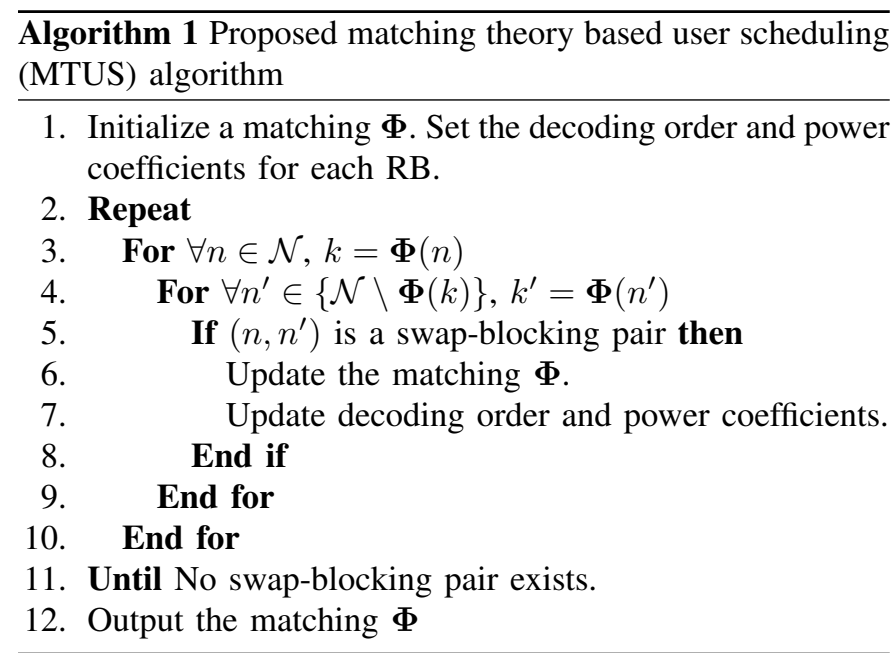

swap operations, all swap-blocking pairs are tackled and the matching achieves the two-sided exchange-stable (2ES).

\section{B. MTUS Algorithm}

In this subsection we propose the MTUS algorithm according to the definitions introduced above. The proposed algorithm searches the swap-blocking pair in the current matching and approves the swap matching until a 2ES matching is obtained.

Before the swap operations, an initial solution should be generated. For simplicity, we pair user $n$ with user $2 K-n+$ 1 , i.e., the strongest user with the weakest user, for fairness. Moreover, the user with lower channel gain is allocated with the larger power coefficient and will be decoded first according to the optimal scheduling for NOMA with perfect SIC [25].

After the initialization, the BS checks all possible pairs of users to search the swap-blocking pair. If a swap-blocking pair is found, the BS swaps the users and updates the corresponding decoding order and power coefficients to improve the system performance. If no swap-blocking pair can be found, the final 2ES matching is obtained. The proposed algorithm can be summarized in Algorithm 1.

For the proposed matching theory based algorithm, we have the following proposition on the complexity.

Proposition 1. The complexity of the proposed MTUS algorithm is upper bounded by $O\left(N^{4}\right)$.

Proof. The complexity of proposed MTUS algorithm is mainly determined by the number of iterations and tests of swap matching. Duplicate swaps between a pair of users is avoided since the swap matching is approved with strictly increase of performance. Therefore, the maximum number of swap operations is $O\left(N^{2}\right)$, which limits the number of iterations to $O\left(N^{2}\right)$. For one iteration, the number of tests for each user is limited by $O(N)$. Therefore, the maximum number of tests for one iteration is $O\left(N^{2}\right)$. In summary, the complexity of the proposed MTUS algorithm is upper bounded by $O\left(N^{4}\right)$.

The complexity of the exhaustive search method increases exponentially with the number of users. Therefore, the com- plexity of the proposed MTUS algorithm is much lower than the exhaustive search method.

\section{Power Allocation}

After user scheduling is performed, we need to allocate transmit powers for NOMA users sharing the same RB. Since each user has been allocated with a fixed RB and a fixed decoding order after performing the MTUS algorithm, we can write $P_{\pi_{k}(m)}=P_{k, m}, P_{\pi_{k}(m)}^{\text {out }}=P_{k, m}^{\text {out }}, \lambda_{\pi_{k}(m)}=$ $\lambda_{k, m}, \phi_{\pi_{k}(m)}=\phi_{k, m}$, and $\epsilon_{\pi_{k}(m)}=\epsilon_{k, m}$ without loss of generality. The power allocation problem can be formulated as

$$
\begin{aligned}
& \text { (P3) } \min _{\left\{P_{k, m}\right\}} \sum_{\forall k, m} P_{k, m} \\
& \text { s.t. } \quad P_{k, m}^{\text {out }} \leq \epsilon_{k, m}, \\
& P_{k, m} \geq 0, \forall k, m \text {. }
\end{aligned}
$$

The problem that minimizes the transmit power subject to the traditional outage probability constraints has been investigated in [25]. However, perfect SIC is assumed in [25] so that the algorithm cannot be applied to solve the problem $(\mathbf{P 3})$. In order to handle the influence of imperfect SIC, we propose a BB based optimal algorithm for power allocation with the traditional outage probability. The more details can be found in Section IV-A. For the alternative outage probability, we can observe that its expression in (8) contains the difference of exponential functions, which makes it more difficult to obtain an optimal solution. Therefore, we apply the CCCP method to find a suboptimal solution for power allocation with alternative outage probability. The more details can be found in Section IV-B.

\section{A. Power Allocation with Traditional Outage Probability}

We first employ the traditional outage probability in the outage constraints. The problem $(\mathbf{P 3})$ can be written as

$$
\begin{aligned}
\min _{\left\{P_{k, 1}, P_{k, 2}\right\}} & \sum_{\forall k}\left(P_{k, 1}+P_{k, 2}\right) \\
\text { s.t. } \quad & 1-e^{-\frac{\phi_{k, 1}}{\rho \lambda_{k, 1}\left(P_{k, 1}-\phi_{k, 1} P_{k, 2}\right)}} \leq \epsilon_{k, 1}, \\
& 1-e^{-\frac{\phi_{k, 1}}{\rho \lambda_{k, 2}\left(P_{k, 1}-\phi_{k, 1} P_{k, 2}\right)}}+g_{k}\left(P_{k, 1}, P_{k, 2}\right) \leq \epsilon_{k, 2}, \\
& P_{k, 1} \geq 0, P_{k, 2} \geq 0, \forall k .
\end{aligned}
$$

We can easily observe that the constraint (17c) can be equally converted to the following two constraints,

$$
1-e^{-\frac{\phi_{k, 1}}{\rho \lambda_{k, 2}\left(P_{k, 1}-\phi_{k, 1} P_{k, 2}\right)}} \leq \epsilon_{k, 2},
$$

and

$$
1-\frac{P_{k, 2}}{P_{k, 2}+P_{k, 1} \omega \phi_{k, 2}} e^{-\frac{\phi_{k, 2}}{\rho \lambda_{k, 2} P_{k, 2}}} \leq \epsilon_{k, 2} .
$$

It is worth noting that power allocation for each $\mathrm{RB}$ is independent for fixed user scheduling and can be decoupled into $K$ subproblems. Therefore, we choose the power allocation for $\mathrm{RB} k$ as the typical one to analyze. Without loss of generality, we can omit the RB index $k$ in subsequent discussion. 
After a series of transformations, the power allocation problem for a typical RB can be expressed as

$$
\text { (P4) } \begin{aligned}
\min _{P_{1}, P_{2}} & P_{1}+P_{2} \\
\text { s.t. } \quad & P_{1}-\phi_{1} P_{2} \geq-\frac{\phi_{1}}{\rho \lambda_{1} \log \left(1-\epsilon_{1}\right)}, \\
& P_{1}-\phi_{1} P_{2} \geq-\frac{\phi_{1}}{\rho \lambda_{2} \log \left(1-\epsilon_{2}\right)}, \\
& 1-\frac{P_{2}}{P_{2}+P_{1} \omega \phi_{2}} e^{-\frac{\phi_{2}}{\rho \lambda_{2} P_{2}}} \leq \epsilon_{2}, \\
& P_{1} \geq 0, P_{2} \geq 0 .
\end{aligned}
$$

The non-convex constraint (20d) makes the problem nonconvex and difficult to solve. However, with the help of the BB technique introduced in [32] and [36], we can still develop an algorithm to obtain an optimal solution.

Before using the BB technique, we should first convert the problem to the standard BB form. By introducing the auxiliary variable $t=e^{-\frac{\phi_{2}}{\rho \lambda_{2} P_{2}}},(\mathbf{P 4})$ can be equivalently transformed into the following problem

$$
\text { (P5) } \begin{aligned}
\min _{t, P_{1}, P_{2}} & -\frac{\left(1+\phi_{1}\right) \phi_{2}}{\rho \lambda_{2} \log (t)}+\beta \\
\text { s.t. } \quad & P_{1}=\phi_{1} P_{2}+\beta, \\
& t=e^{-\frac{\phi_{2}}{\rho \lambda_{2} P_{2}}}, \\
& 1-\frac{P_{2}}{P_{2}+P_{1} \omega \phi_{2}} t \leq \epsilon_{2}, \\
& P_{1} \geq 0, P_{2} \geq 0,
\end{aligned}
$$

where $\beta=\max \left(-\frac{\phi_{1}}{\rho \lambda_{1} \log \left(1-\epsilon_{1}\right)},-\frac{\phi_{1}}{\rho \lambda_{2} \log \left(1-\epsilon_{2}\right)}\right)$. The proof of equivalence between (P4) and (P5) is provided in Appendix A.

If we denote the objective function and feasible set of $t$ for the optimization problem $(\mathbf{P 5})$ as $\mathcal{L}(t)$ and $\Psi$, respectively, the problem can be converted to

$$
\begin{array}{cll}
\text { (P6) } & \min _{t} & \mathcal{L}(t) \\
& \text { s.t. } & t \in \Psi .
\end{array}
$$

By observing the problem $(\mathbf{P 5})$, we can find $0<t<1$. Therefore, the objective function $\mathcal{L}(t)$ is a non-decreasing function. As a result, if we find a feasible point $t_{0}$, the feasible set can be reduced to $\Psi^{\prime}=\left\{t \mid t \in \Psi, t \leq t_{0}\right\}$ without affecting the optimality. In order to formulate the standard form for the BB method, we define the following extended function

$$
\tilde{\mathcal{L}}(t)= \begin{cases}\mathcal{L}(t), & \text { if } t \in \Psi^{\prime}, \\ \mathcal{L}\left(t_{0}\right), & \text { otherwise. }\end{cases}
$$

If we define the function

$$
f(t \mid \Pi)=\inf _{t \in \Pi} \tilde{\mathcal{L}}(t),
$$

which is the infimum of function $\tilde{\mathcal{L}}(t)$ in the feasible set $\Pi$, we can have the following relationship

$$
f(t \mid \Pi)=f\left(t \mid \Psi^{\prime}\right)=p^{\star},
$$

where $\Psi^{\prime} \subseteq \Pi$ and $p^{\star}$ denotes optimal objective function value. From constraint (21d), the variable $t$ is lower bounded by $1-\epsilon_{2}$. Therefore, the extended feasible set can be chosen as $\Pi=\left\{t \mid 1-\epsilon_{2} \leq t \leq t_{0}\right\}$. Furthermore, the problem (P6) can be converted to minimizing the function $\tilde{\mathcal{L}}(t)$ over the extended set $\Pi$.

The BB method is to iteratively divide the feasible set $\Pi$ into smaller subsets for searching, which is organized as a binary tree. At each leaf node, we can calculate the upper bound and lower bound for the function $f$. By removing the leaf nodes whose lower bounds are larger than the minimum value over all upper bounds, the gap between the upper and lower bound is reduced. By repeating the procedure until the accuracy requirement is satisfied, a global optimal solution will be obtained.

By utilizing the monotonic increasing feature of function $\mathcal{L}(t)$, we can refer to [32] and construct the lower bound function for the set $\Pi=\{t \mid \underline{t} \leq t \leq \bar{t}\}$ as

$$
\underline{f}(t \mid \Pi)= \begin{cases}\mathcal{L}(\underline{t}), & \text { if } \bar{t} \in \Psi^{\prime}, \\ \mathcal{L}\left(t_{0}\right), & \text { otherwise, }\end{cases}
$$

and the upper bound function as

$$
\bar{f}(t \mid \Pi)= \begin{cases}\mathcal{L}(\bar{t}), & \text { if } \bar{t} \in \Psi^{\prime} \\ \mathcal{L}\left(t_{0}\right), & \text { otherwise }\end{cases}
$$

The key step to calculate the bound function is to check whether $\bar{t} \in \Psi^{\prime}$. By considering the constraints (21b) and (21c), we set

$$
P_{2}=-\frac{\phi_{2}}{\rho \lambda_{2} \log (\bar{t})},
$$

and

$$
P_{1}=-\frac{\phi_{1} \phi_{2}}{\rho \lambda_{2} \log (\bar{t})}+\beta .
$$

If the constraint (21d) is satisfied with (28), (29), and $t=\bar{t}$, the condition $\bar{t} \in \Psi^{\prime}$ is guaranteed.

Based on the above definitions and discussions, we can describe the proposed power allocation algorithm as follows. First, we calculate a feasible point $t_{0}$ based on Algorithm 2 and initialize the feasible set as $\Pi_{1}=\left\{t \mid 1-\epsilon_{2} \leq t \leq t_{0}\right\}$. For the $i$-th iteration, we divide the feasible set $\Pi_{i}$ into two subsets $\Pi_{i, 1}$ and $\Pi_{i, 2}$ and calculate the corresponding lower and upper bounds, respectively. Due to the monotonic increasing feature of the function $\mathcal{L}(t)$, there will always be a subset whose lower bound is greater than or equal to the upper bound of the other one. By removing the subset with a larger lower bound, we can reduce the search space without affecting the optimality. When the gap between the lower bound and upper bound is small enough, the iteration stops and a global optimal solution is obtained. The proposed algorithm is summarized in Algorithm 3.

The calculations in Algorithm 2 and Algorithm 3 are scalar based. Therefore, the complexity of the proposed algorithm for a typical RB can be denoted as $O\left(I_{1}+I_{2}\right)$, where $I_{1}$ and $I_{2}$ denote number of iterations in Algorithm 2 and Algorithm 3, respectively. There are $K$ RBs so that the total complexity of the proposed algorithm can be expressed as $O\left(K\left(I_{1}+I_{2}\right)\right)$. 

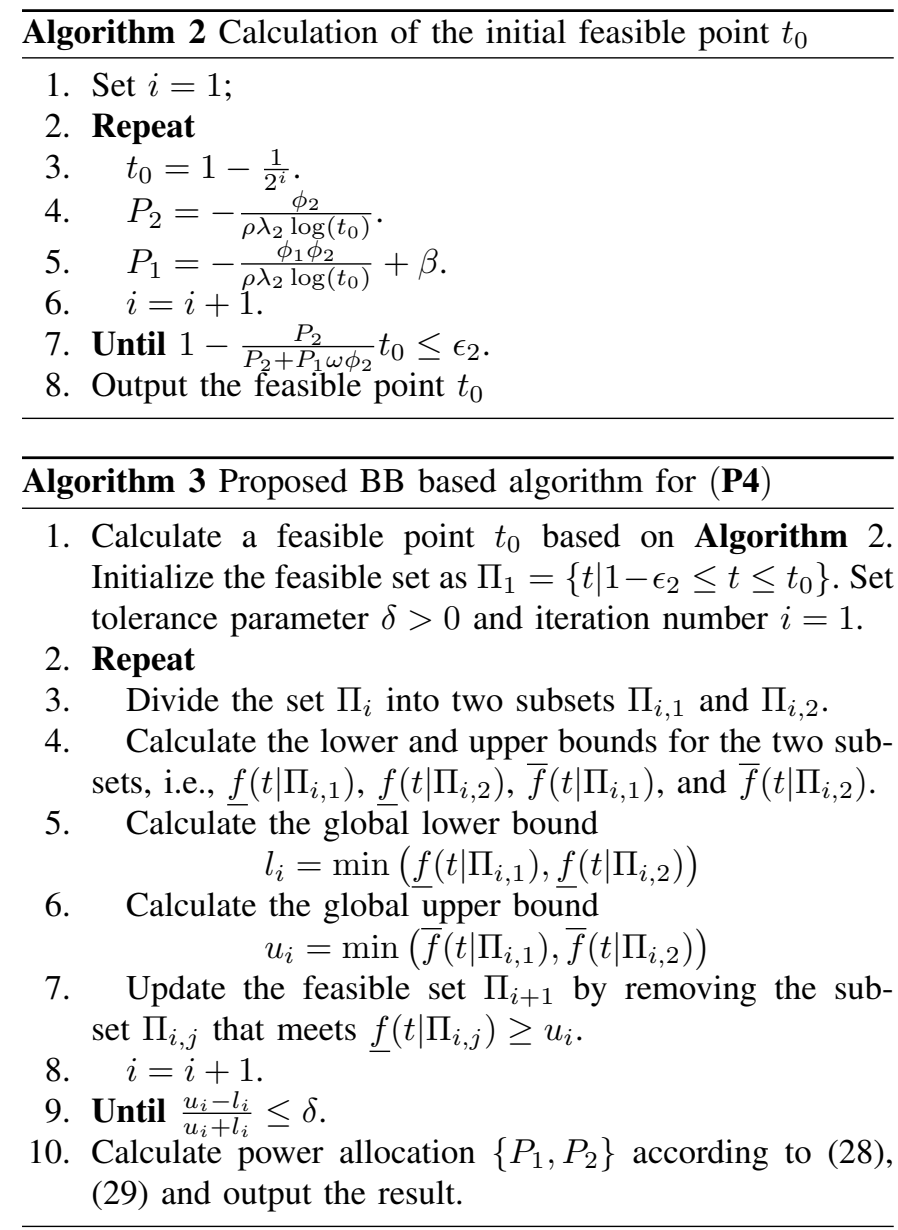

\section{B. Power Allocation with Alternative Outage Probability}

By employing the alternative outage probability in the outage constraints, $(\mathbf{P 3})$ can be rewritten as

$$
\begin{aligned}
\min _{\left\{P_{k, 1}, P_{k, 2}\right\}} & \sum_{\forall k}\left(P_{k, 1}+P_{k, 2}\right) \\
\text { s.t. } \quad & 1-e^{-\frac{\phi_{k, 1}}{\rho \lambda_{k, 1}\left(P_{k, 1}-\phi_{k, 1} P_{k, 2}\right)}} \leq \epsilon_{k, 1}, \\
& 1-e^{-\min \left(\frac{\phi_{k, 1}}{\rho \lambda_{k, 2}\left(P_{k, 1}-\phi_{k, 1} P_{k, 2}\right)}, \frac{\phi_{k, 2}}{\rho \lambda_{k, 2}\left(P_{k, 2}-\phi_{k, 2} P_{k, 1}\right)}\right)} \\
& +g_{k}\left(P_{k, 1}, P_{k, 2}\right) \leq \epsilon_{k, 2}, \\
& P_{k, 1} \geq 0, P_{k, 2} \geq 0, \forall k .
\end{aligned}
$$

Similar to the scheme with the traditional outage probability, power allocation for each RB can also be decoupled. Hence, we omit the RB index and formulate the power allocation problem for a typical $\mathrm{RB}$ as

$$
\begin{array}{ll}
\min _{P_{1}, P_{2}} & P_{1}+P_{2} \\
\text { s.t. } \quad & P_{1}-\phi_{1} P_{2} \geq-\frac{\phi_{1}}{\rho \lambda_{1} \log \left(1-\epsilon_{1}\right)}, \\
& 1-e^{-\min \left(\frac{\phi_{1}}{\rho \lambda_{2}\left(P_{1}-\phi_{1} P_{2}\right)}, \frac{\phi_{2}}{\rho \lambda_{2}\left(P_{2}-\phi_{2} P_{1}\right)}\right)}+g\left(P_{1}, P_{2}\right) \leq \epsilon_{2}, \\
& P_{1} \geq 0, P_{2} \geq 0 .
\end{array}
$$

As discussed in the previous section, the alternative outage probability is different from the traditional one only when $\frac{\phi_{2}}{\left(P_{2}-\phi_{2} P_{1}\right)}<\frac{\phi_{1}}{\left(P_{1}-\phi_{1} P_{2}\right)}$. Therefore, we can divide (P7) into two subproblems to handle the complicated term $e^{-\min \left(\frac{\phi_{1}}{\rho \lambda_{2}\left(P_{1}-\phi_{1} P_{2}\right)}, \frac{\phi_{2}}{\rho \lambda_{2}\left(P_{2}-\phi_{2} P_{1}\right)}\right)}$ in constraint (31c). The first subproblem is to optimize power allocation with the constraint $\frac{\phi_{2}}{\left(P_{2}-\phi_{2} P_{1}\right)} \geq \frac{\phi_{1}}{\left(P_{1}-\phi_{1} P_{2}\right)}$, which is similar to $(\mathbf{P 4})$ and can be solved via the $\mathrm{BB}$ method. The second subproblem is with the constraint $\frac{\phi_{2}}{\left(P_{2}-\phi_{2} P_{1}\right)}<\frac{\phi_{1}}{\left(P_{1}-\phi_{1} P_{2}\right)}$ and can be formulated as

$$
\text { (P8) } \begin{aligned}
\min _{P_{1}, P_{2}} & P_{1}+P_{2} \\
\text { s.t. } \quad & (31 \mathrm{~b}),(31 \mathrm{~d}), \\
& 1-e^{-\frac{\phi_{2}}{\rho \lambda_{2}\left(P_{2}-\phi_{2} P_{1}\right)}}+g\left(P_{1}, P_{2}\right) \leq \epsilon_{2}, \\
& \phi_{2}\left(P_{1}-\phi_{1} P_{2}\right) \leq \phi_{1}\left(P_{2}-\phi_{2} P_{1}\right) .
\end{aligned}
$$

By comparing the solutions for the two subproblems and choose the one that leads to lower objective function value, we can obtain the solution of the original problem $(\mathbf{P 7})$. Note that we can omit the constraint $\frac{\phi_{2}}{\left(P_{2}-\phi_{2} P_{1}\right)} \geq \frac{\phi_{1}}{\left(P_{1}-\phi_{1} P_{2}\right)}$ for the first subproblem without influencing the optimality. If the solution fall within the area $\frac{\phi_{2}}{\left(P_{2}-\phi_{2} P_{1}\right)}<\frac{\phi_{1}}{\left(P_{1}-\phi_{1} P_{2}\right)}$, the alternative outage constraints will lead to better performance, which indicates that the solution of the second subproblem is better.

Constraint (32c) is highly non-convex, which makes it difficult to obtain an optimal solution for subproblem (P8). Therefore, we propose an iterative algorithm based on the CCCP technique, which monotonically improves the performance and converges to a stationary solution [33]. Before applying the CCCP method, we first transform the complicated constraint in (32c) to a more tractable yet equivalent form. By introducing auxiliary variables $\left\{\theta_{1}, \theta_{2}, \tilde{t}_{1}, \tilde{t}_{2}, \bar{t}_{2}, t_{2}\right\},(\mathbf{P 8})$ can be equivalently converted to the following problem

$$
\text { (P9) } \begin{array}{cl}
\min _{\mathcal{Z}} & P_{1}+P_{2} \\
\text { s.t. } & (31 \mathrm{~b}),(31 \mathrm{~d}),(32 \mathrm{~d}), \\
& 1-e^{-\theta_{1}}+\theta_{2} \leq \epsilon_{2}, \\
& \theta_{1} \geq \frac{\phi_{2}}{\rho \lambda_{2}\left(P_{2}-\phi_{2} P_{1}\right)}, \\
& \theta_{2} \geq 0, \theta_{2} \geq e^{-\tilde{t}_{1}}-t_{2}, \\
& \tilde{t}_{1} \leq \frac{\phi_{1}}{\rho \lambda_{2}\left(P_{1}-\phi_{1} P_{2}\right)}, \\
& \tilde{t}_{2} \geq \frac{\phi_{2}}{\rho \lambda_{2} P_{2}}, \\
& \bar{t}_{2} \leq e^{-\tilde{t}_{2}}, \\
& t_{2} \leq \frac{P_{2}}{P_{2}+P_{1} \omega \phi_{2}} \bar{t}_{2},
\end{array}
$$

where $\mathcal{Z} \triangleq\left\{P_{1}, P_{2}, \theta_{1}, \theta_{2}, \tilde{t}_{1}, \tilde{t}_{2}, \bar{t}_{2}, t_{2}\right\}$ denotes the set of variables. The proof of equivalence between (P8) and (P9) is provided in Appendix B. After the transformation, the highly coupled non-convex constraint (32c) is divided into several simpler constraints (33c)-(33i), which are easier to handle. However, some of the constraints are still non-convex, which complicate the problem, such as (33c), (33f), (33h), and (33i). 
Algorithm 4 Proposed CCCP-based algorithm for the problem (P8)

1. Initialize a feasible point $\mathcal{Z}$. Set the tolerance of accuracy $\delta$ and iteration number $i=0$.

2. Repeat

3. Update the variables by solving the problem $(\mathbf{P 1 0})$ via CVX.

4. Update the iteration number : $i=i+1$.

5. Until $\frac{\left|\mathcal{L}^{i+1}(\mathcal{Z})-\mathcal{L}^{i}(\mathcal{Z})\right|}{\left|\mathcal{L}^{i}(\mathcal{Z})\right|} \leq \delta$.

6. Output the solution $\left\{P_{1}, P_{2}\right\}$

We can write the non-convex constraints as difference-ofconvex (DC) forms and then approximate them as convex constraints through the first-order Taylor expansion. By taking the non-convex constraint (33i) as an example, we then rewrite (33i) into the DC form in (34). By applying the first-order Taylor expansion to linearize the subtracted convex functions, an approximated convex constraint can be obtained as in (35), where $P_{1}^{i}, P_{2}^{i}, t_{2}^{i}$, and $\bar{t}_{2}^{i}$ denote the current points of the variables $P_{1}, P_{2}, t_{2}$, and $\bar{t}_{2}$, respectively. Furthermore, the approximated convex constraint can be written as the secondorder cone (SOC) form in (36). By applying the same method, the non-convex constraints (33c), (33f), and (33h) can be approximated as the convex ones in (37)-(39), respectively.

$$
1-\epsilon_{2}+e^{-\theta_{1}^{i}}\left(\theta_{1}-\theta_{1}^{i}-1\right)+\theta_{2} \leq 0,
$$

and

$$
\bar{t}_{2}+e^{-\tilde{t}_{2}^{i}}\left(\tilde{t}_{2}-\tilde{t}_{2}^{i}-1\right) \leq 0 .
$$

Moreover, the constraints (33d) and (33g) can be reformulated as the following SOC constraints

$$
\left\|\left[\sqrt{\frac{\phi_{2}}{\rho \lambda_{2}}}, \frac{\left(\theta_{1}-\left(P_{2}-\phi_{2} P_{1}\right)\right)}{2}\right]\right\| \leq \frac{\left(\theta_{1}+\left(P_{2}-\phi_{2} P_{1}\right)\right)}{2},
$$

and

$$
\left\|\left[\sqrt{\frac{\phi_{2}}{\rho \lambda_{2}}}, \frac{\left(\tilde{t}_{2}-P_{2}\right)}{2}\right]\right\| \leq \frac{\left(\tilde{t}_{2}+P_{2}\right)}{2} .
$$

Finally, we obtain the approximated convex problem for $(\mathbf{P 9})$ in the $i$-th iteration expressed as

$$
\begin{aligned}
\text { (P10) } \min _{\mathcal{Z}} & P_{1}+P_{2} \\
\text { s.t. } & (31 \mathrm{~b}),(31 \mathrm{~d}),(32 \mathrm{~d}),(33 \mathrm{e}), \\
& (36)-(41) .
\end{aligned}
$$

We can solve the convex problem (P10) via the convex programming toolbox CVX [37] in each iteration. The CCCP based iterative algorithm is summarized in Algorithm 4, where $\mathcal{L}(\mathcal{Z})$ denotes the objective function value.

By combining the optimization algorithms for the two subproblems, we can obtain the overall power allocation algorithm for the alternative outage probability summarized in Algorithm 5.

For a typical RB, the complexity of solving the first subproblem (P4) has been analyzed in the previous subsection,

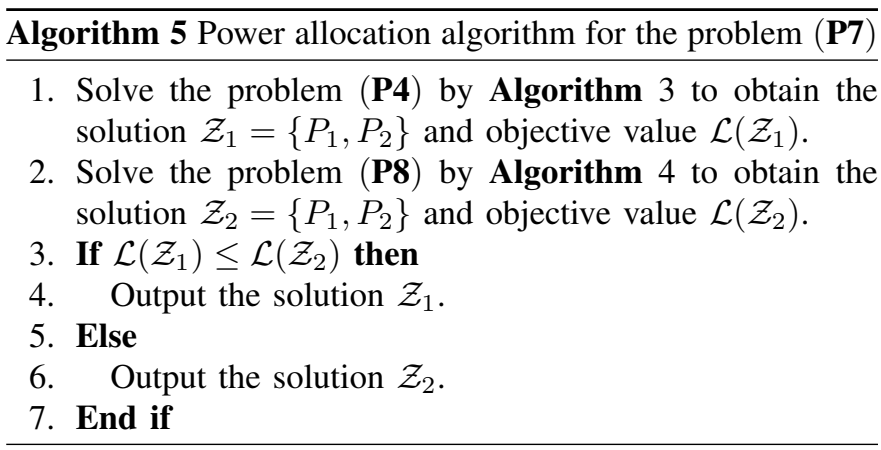

which can be expressed as $O\left(I_{1}+I_{2}\right)$. The variables in the CCCP based algorithm for the second subproblem $(\mathbf{P 8})$ are scalars. Therefore, the complexity of Algorithm 4 can be expressed as $O\left(I_{3}\right)$, where $I_{3}$ denotes the number of CCCP iterations. Based on the complexity analyses for the two subproblems, we can denote the complexity of the overall algorithm for (P7) summarized in Algorithm 5 as $O\left(I_{1}+I_{2}+I_{3}\right)$. For the system with $K$ RBs and $2 K$ users, the total complexity of power allocation can be expressed as $O\left(K\left(I_{1}+I_{2}+I_{3}\right)\right)$.

\section{Simulation Results}

In this section, we evaluate the performance of the proposed algorithm through simulation. We assume that $2 K$ users are divided into $K$ orthogonal pairs and assigned to $K$ RBs. Moreover, we set $K=3$ generally. The users are uniformly distributed within a circle with radius $r_{c}=10 \mathrm{~m}$. For simplicity, the noise power is normalized and set as $\sigma^{2}=0 \mathrm{~dB}$. The frequency dependent factor and the path loss exponent are set as $\eta=1$ and $\alpha=2$. Moreover, we assume that all users have the same QoS constraint, i.e., $R_{n}=R$ and $\epsilon_{n}=\epsilon$ for $n \in \mathcal{N}$. The fixed power coefficients used in the MTUS algorithm are chosen as $P_{k, a}=10 \lg \left(-\frac{\phi}{\lambda \log (1-\epsilon)}\right) \mathrm{dB}$ and $P_{k, b}=$ $10 \lg \left(-\frac{\phi}{2 \lambda \log (1-\epsilon)}\right) \mathrm{dB}$ for $k \in \mathcal{K}$, where $\phi=2^{R}-1$ and $\lambda=\frac{1}{2 K} \sum_{n=1}^{2 K} \lambda_{n}$. The tolerance accuracy of the iterative algorithm is set as $\delta=10^{-6}$.

Fig. 1 and Fig. 2 show the convergence performance of the proposed $\mathrm{BB}$ and CCCP based algorithms, respectively. The users are randomly paired. The target rate is set as $R=$ $0.1 \mathrm{bits} / \mathrm{s} / \mathrm{Hz}$, and the variance of the residual interference is $\omega=-10 \mathrm{~dB}$. Fig. 1 presents the minimized total transmit power versus the number of iterations for the BB based algorithm summarized in Algorithm 3. From the figure, the gap between the lower bound and upper bound becomes smaller with the increase of iteration numbers. Fig. 2 presents the minimized total transmit power versus the number of iterations for the CCCP based algorithm summarized in Algorithm 4 . From the figure, the minimized total transmit power decreases with the increase of iteration numbers and converges finally. Moreover, the algorithm converges fast with loose outage probability constraint.

Fig. 3 illustrates the required transmit power versus the outage probability constraint for different user scheduling algorithms. The variance of the residual interference is assumed as $\omega=-10 \mathrm{~dB}$, and the target rate is set as $R=0.1 \mathrm{bits} / \mathrm{s} / \mathrm{Hz}$. We mainly consider the following algorithms for comparison: 


$$
\begin{aligned}
& \frac{\left(P_{2}+\left(t_{2}-\bar{t}_{2}\right)\right)^{2}}{4}+\omega \phi_{2} \frac{\left(P_{1}-t_{2}\right)^{2}}{4}-\frac{\left(P_{2}-\left(t_{2}-\bar{t}_{2}\right)\right)^{2}}{4}-\omega \phi_{2} \frac{\left(P_{1}+t_{2}\right)^{2}}{4} \leq 0 . \\
& \frac{\left(P_{2}+\left(t_{2}-\bar{t}_{2}\right)\right)^{2}}{4}+\omega \phi_{2} \frac{\left(P_{1}-t_{2}\right)^{2}}{4}-\left(\frac{\left(P_{2}^{i}-\left(t_{2}^{i}-\bar{t}_{2}^{i}\right)\right)\left(P_{2}-\left(t_{2}-\bar{t}_{2}\right)\right)}{2}-\frac{\left(P_{2}^{i}-\left(t_{2}^{i}-\bar{t}_{2}^{i}\right)\right)^{2}}{4}\right) \\
& -\omega \phi_{2}\left(\frac{\left(P_{1}^{i}+t_{2}^{i}\right)\left(P_{1}+t_{2}\right)}{2}-\frac{\left(P_{1}^{i}+t_{2}^{i}\right)^{2}}{4}\right) \leq 0 . \\
& \|\left[\frac{\left(P_{2}+\left(t_{2}-\bar{t}_{2}\right)\right)}{2}, \sqrt{\omega \phi_{2}} \frac{\left(P_{1}-t_{2}\right)}{2}, \frac{\left(P_{2}^{i}-\left(t_{2}^{i}-\bar{t}_{2}^{i}\right)\right)\left(P_{2}-\left(t_{2}-\bar{t}_{2}\right)\right)}{4}-\frac{\left(P_{2}^{i}-\left(t_{2}^{i}-\bar{t}_{2}^{i}\right)\right)^{2}}{8}\right. \\
& \left.+\omega \phi_{2}\left(\frac{\left(P_{1}^{i}+t_{2}^{i}\right)\left(P_{1}+t_{2}\right)}{4}-\frac{\left(P_{1}^{i}+t_{2}^{i}\right)^{2}}{8}\right)-\frac{1}{2}\right] \| \leq \frac{\left(P_{2}^{i}-\left(t_{2}^{i}-\bar{t}_{2}^{i}\right)\right)\left(P_{2}-\left(t_{2}-\bar{t}_{2}\right)\right)}{4} \\
& -\frac{\left(P_{2}^{i}-\left(t_{2}^{i}-\bar{t}_{2}^{i}\right)\right)^{2}}{8}+\omega \phi_{2}\left(\frac{\left(P_{1}^{i}+t_{2}^{i}\right)\left(P_{1}+t_{2}\right)}{4}-\frac{\left(P_{1}^{i}+t_{2}^{i}\right)^{2}}{8}\right)+\frac{1}{2} . \\
& \left\|\left[\frac{\left(\tilde{t}_{1}+\left(P_{1}-\phi_{1} P_{2}\right)\right)}{2}, \frac{\left(\tilde{t}_{1}^{i}-\left(P_{1}^{i}-\phi_{1} P_{2}^{i}\right)\right)\left(\tilde{t}_{1}-\left(P_{1}-\phi_{1} P_{2}\right)\right)}{4}-\frac{\left(\tilde{t}_{1}^{i}-\left(P_{1}^{i}-\phi_{1} P_{2}^{i}\right)\right)^{2}}{8}+\frac{\phi_{1}}{2 \rho \lambda_{2}}-\frac{1}{2}\right]\right\| \\
& \leq \frac{\left(\tilde{t}_{1}^{i}-\left(P_{1}^{i}-\phi_{1} P_{2}^{i}\right)\right)\left(\tilde{t}_{1}-\left(P_{1}-\phi_{1} P_{2}\right)\right)}{4}-\frac{\left(\tilde{t}_{1}^{i}-\left(P_{1}^{i}-\phi_{1} P_{2}^{i}\right)\right)^{2}}{8}+\frac{\phi_{1}}{2 \rho \lambda_{2}}+\frac{1}{2} .
\end{aligned}
$$

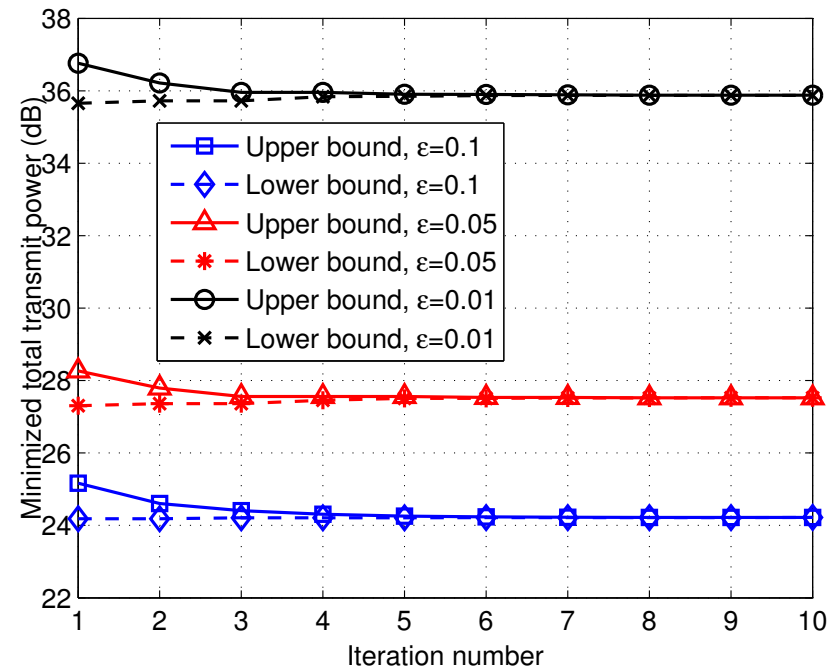

Fig. 1: The convergence performance of the proposed BB based algorithm: minimized total transmit power versus the number of iterations.

- Exhaust: optimize power allocation for all possible user scheduling and choose the scheme with minimum power.

- MTUS: apply the MTUS algorithm to obtain user scheduling and then optimize power allocation.

- Random: randomly choose user scheduling and then optimize power allocation.

Here AL and TR denote the schemes based on the alternative outage probability and traditional outage probability, respectively. Note that the exhaust algorithm optimizes power

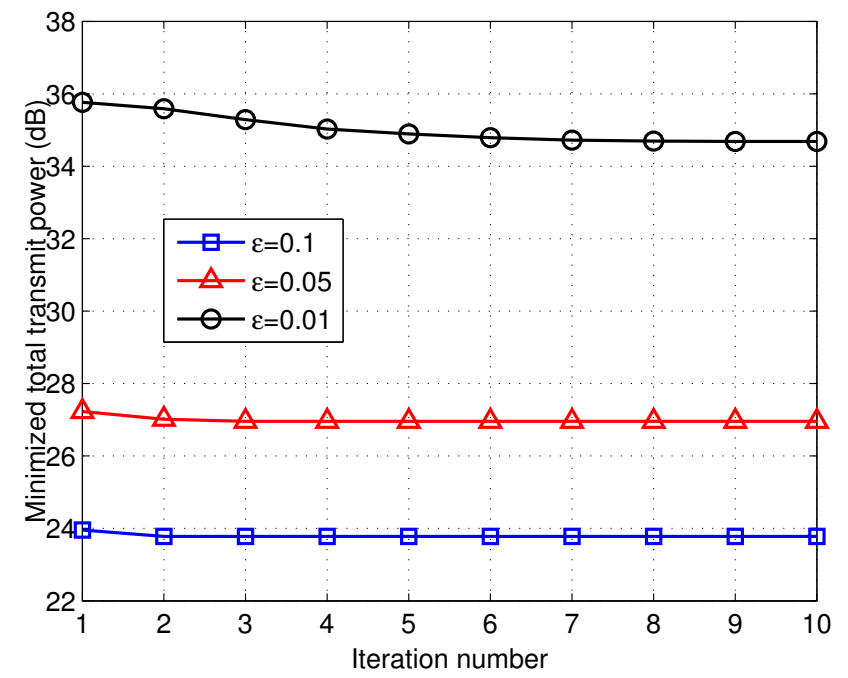

Fig. 2: The convergence performance of the proposed CCCP based algorithm: minimized total transmit power versus the number of iterations.

allocation for all candidate user scheduling, which results in extremely high complexity. In the MTUS algorithm, power allocation is only optimized once so that the complexity is much lower. However, the MTUS algorithm can still achieve near-optimal performance as shown in the figure. Moreover, the alternative scheme with MTUS algorithm can even outperform the traditional scheme with exhaust algorithm under strict outage constraints.

Fig. 4 compares the required transmit power versus the 


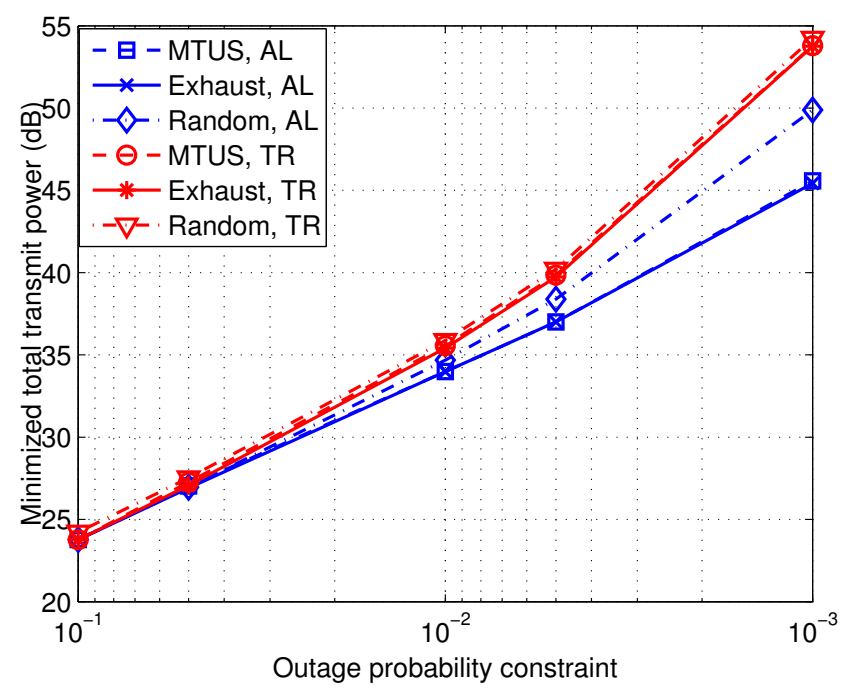

Fig. 3: Minimized total transmit power versus outage probability constraint $\epsilon$, with $\omega=-10 \mathrm{~dB}$ and $R=0.1 \mathrm{bits} / \mathrm{s} / \mathrm{Hz}$.

outage probability constraint with different target rates for the algorithms based on the traditional outage probability and the alternative one. The variance of the residual interference is $\omega=-10 \mathrm{~dB}$. From the figure, the gap between two schemes becomes larger with the decreasing outage probability constraint. That is to say, the superiority of the algorithm based on alternative outage probability is more significant when the tolerance of outage is lower. The reason is that the residual interference only affects the user to be decoded second in NOMA and becomes the major limiting factor for the performance when the outage probability constraint is low. Hence, the required power for the user to be decoded second can be larger than that for the user to be decoded first. As a result, the condition $\frac{\phi_{\pi_{k}(2)}}{\left(P_{\pi_{k}(2)}-\phi_{\pi_{k}(2)} P_{\left.\pi_{k}(1)\right)}\right.}<\frac{\phi_{\pi_{k}(1)}}{\left(P_{\pi_{k}(1)}-\phi_{\pi_{k}(1)} P_{\left.\pi_{k}(2)\right)}\right.}$ is more likely to achieve, which indicates that the algorithm based on the alternative outage probability has better performance. Moreover, with higher target rate, the minimized total transmit power is higher as expected and the gain of the algorithm based on the alternative outage probability is also larger.

Fig. 5 compares the required transmit power versus the variance of residual interference with different target rates for the algorithms based on the traditional outage probability and the alternative one. The outage probability constraint is set as $\epsilon=0.01$. From the figure, the required transmit power increases with the residual interference variance as expected. Moreover, the performance gap between the two schemes also becomes larger with increasing residual interference.

Fig. 6 compares the required transmit power versus the outage probability constraint with different RB numbers for the algorithms based on the traditional outage probability and the alternative one. The variance of the residual interference is $\omega=-10 \mathrm{~dB}$. From the figure, the required transmit power increases with the number of RBs. However, the algorithm based on the alternative outage probability still achieves better performance and the performance gap between the two

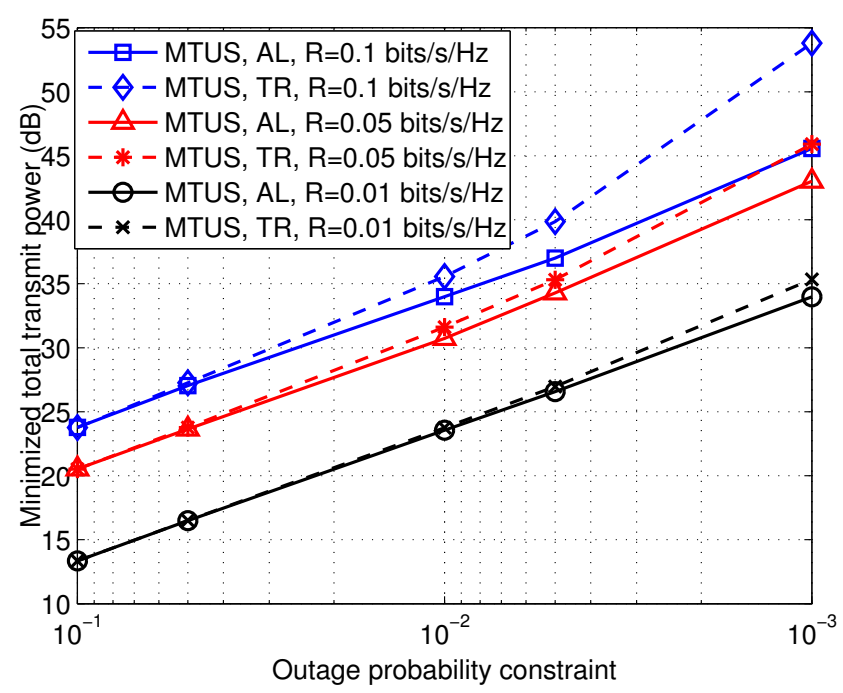

Fig. 4: Minimized total transmit power versus outage probability constraint $\epsilon$, with different target rates and $\omega=-10 \mathrm{~dB}$.

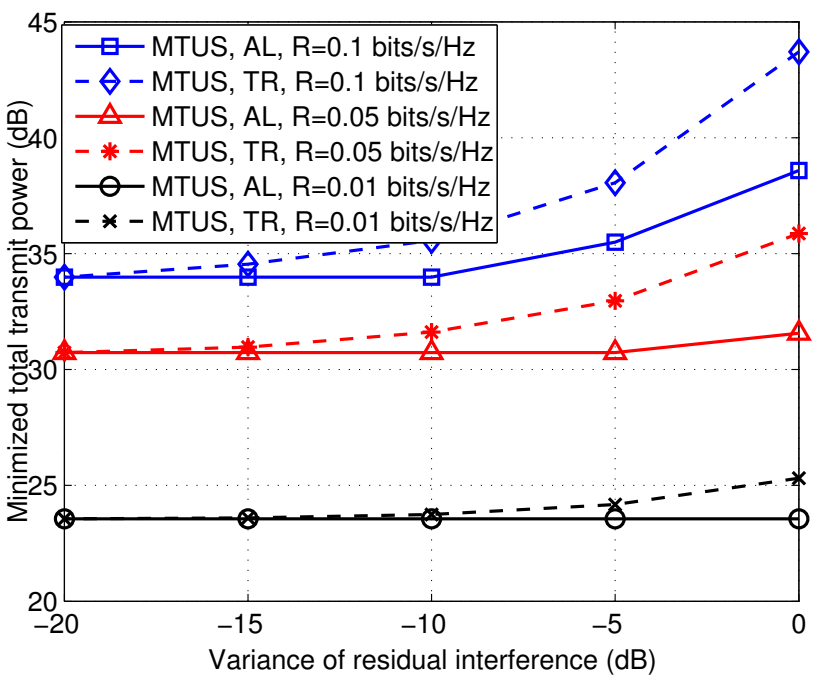

Fig. 5: Minimized total transmit power versus variance of residual interference $\omega$, with different target rates and $\epsilon=0.01$.

schemes is approximately stable.

Fig. 7 compares the required transmit power versus the outage probability constraint for the proposed NOMA and conventional OMA algorithms. The RB number is set as $K=3$ and the target rate is set as $R=0.1 \mathrm{bits} / \mathrm{s} / \mathrm{Hz}$. From the figure, the performance of NOMA degrades when the variance of residual interference increases. Moreover, the impact of imperfect SIC is more significant when the outage probability constraint is smaller. NOMA outperforms OMA when the residual interference caused by imperfect SIC is moderate. If the residual interference is too large, the outage probability of the user to be decoded second in NOMA will be greatly impaired and OMA achieves better performance. 


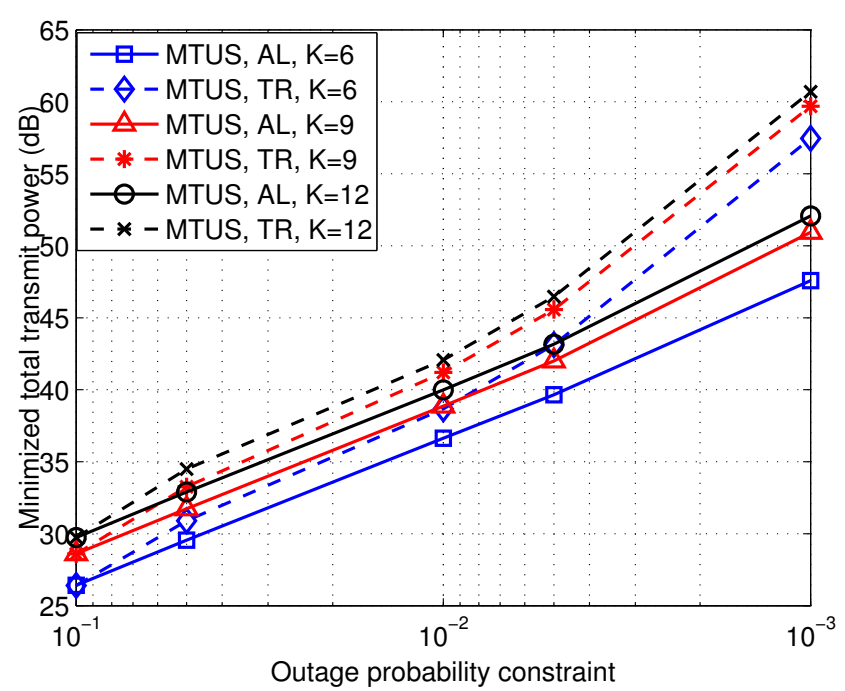

Fig. 6: Minimized total transmit power versus outage probability constraint $\epsilon$, with different RB numbers and $\omega=-10 \mathrm{~dB}$.

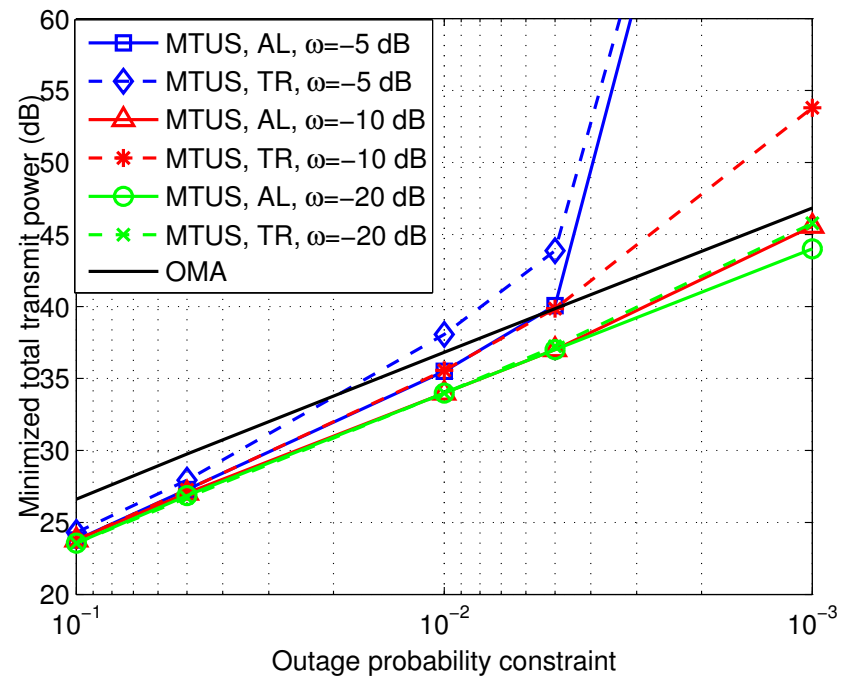

Fig. 7: Minimized total transmit power versus outage probability constraint $\epsilon$, with $K=3$ and $R=0.1$ bits/s/Hz.

\section{CONCLUSION}

In this paper, we have investigated the impact of an alternative outage probability on user scheduling and power allocation for the NOMA system with the imperfect SIC. Specifically, we have minimized the total transmit power where the constraints based on both the traditional outage probability and the alternative one are considered. In order to handle the highly coupled non-convex problem, we have proposed a two-phase algorithm where user scheduling is first addressed via the proposed matching theory based user scheduling algorithm and then power allocation for each pair of NOMA users sharing the same RB is optimized. Simulation results have shown that the proposed algorithm with the alternative outage probability outperforms that of the traditional one when the residual interference from imperfect SIC significantly affects the decoding.

\section{APPENDIX A}

Proof of Equivalence between (P4) And (P5)

By combining the constraints (20b) and (20c), we can equivalently convert them to the following constraint

$$
P_{1} \geq \phi_{1} P_{2}+\beta \text {. }
$$

Moreover, we can observe that the left side of the constraint (20d) is monotonically increasing with $P_{1}$. That is to say, the constraint (20d) is easier to be satisfied if $P_{1}$ is smaller. On the other hand, the objective function of problem $(\mathbf{P 4})$ is to minimize the sum of $P_{1}$ and $P_{2}$. Therefore, we can choose the minimum $P_{1}$, i.e., take the equality in (A.1), without influencing the optimality. As a result, problem (P4) can be equivalently transformed as

$$
\begin{aligned}
\min _{P_{1}, P_{2}} & P_{1}+P_{2} \\
\text { s.t. } & P_{1}=\phi_{1} P_{2}+\beta, \\
& 1-\frac{P_{2}}{P_{2}+P_{1} \omega \phi_{2}} e^{-\frac{\phi_{2}}{\rho \lambda_{2} P_{2}}} \leq \epsilon_{2}, \\
& P_{1} \geq 0, P_{2} \geq 0 .
\end{aligned}
$$

In order to handle the exponential function in the constraint (A.2c), we introduce the auxiliary variable $t=e^{-\frac{\phi_{2}}{\rho \lambda_{2} P_{2}}}$ and rewrite the problem in (A.2) as

$$
\begin{aligned}
\min _{P_{1}, P_{2}, t} & P_{1}+P_{2} \\
\text { s.t. } & P_{1}=\phi_{1} P_{2}+\beta, \\
& t=e^{-\frac{\phi_{2}}{\rho \lambda_{2} P_{2}}}, \\
& 1-\frac{P_{2}}{P_{2}+P_{1} \omega \phi_{2}} t \leq \epsilon_{2}, \\
& P_{1} \geq 0, P_{2} \geq 0 .
\end{aligned}
$$

According to the equality constraints (A.3b) and (A.3c), we can write

$$
\begin{gathered}
P_{2}=-\frac{\phi_{2}}{\rho \lambda_{2} \log (t)}, \\
P_{1}=-\frac{\phi_{1} \phi_{2}}{\rho \lambda_{2} \log (t)}+\beta .
\end{gathered}
$$

By taking (A.4) and (A.5) in the objective function of (A.3), we can obtain $(\mathbf{P 5})$. The proof of equivalence between $(\mathbf{P 4})$ and (P5) is completed.

\section{APPENDIX B}

\section{Proof of Equivalence between (P8) and (P9)}

Let us first introduce variables $\theta_{1}$ and $\theta_{2}$ as the upper bounds of $\frac{\phi_{2}}{\rho \lambda_{2}\left(P_{2}-\phi_{2} P_{1}\right)}$ and $g\left(P_{1}, P_{2}\right)$, respectively. Problem (P8) can be equivalently expressed as

$$
\begin{array}{cl}
\min _{\mathcal{Z}} & P_{1}+P_{2} \\
\text { s.t. } & (31 \mathrm{~b}),(31 \mathrm{~d}),(32 \mathrm{~d}), \\
& 1-e^{-\theta_{1}}+\theta_{2} \leq \epsilon_{2}, \\
& \theta_{1} \geq \frac{\phi_{2}}{\rho \lambda_{2}\left(P_{2}-\phi_{2} P_{1}\right)}, \\
& \theta_{2} \geq 0 \\
& \theta_{2} \geq e^{-\frac{\phi_{1}}{\rho \lambda_{2}\left(P_{1}-\phi_{1} P_{2}\right)}}-\frac{P_{2}}{P_{2}+P_{1} \omega \phi_{2}} e^{-\frac{\phi_{2}}{\rho \lambda_{2} P_{2}}}, .
\end{array}
$$


In order to handle the fraction forms in the exponent in (A.6f), we introduce auxiliary variables $\tilde{t}_{1}$ and $\tilde{t}_{2}$ as the lower bound and upper bound of $\frac{\phi_{1}}{\rho \lambda_{2}\left(P_{1}-\phi_{1} P_{2}\right)}$ and $\frac{\phi_{2}}{\rho \lambda_{2} P_{2}}$, respectively. As a result, the constraint (A.6f) can be equivalently converted to

$$
\begin{gathered}
\tilde{t}_{1} \leq \frac{\phi_{1}}{\rho \lambda_{2}\left(P_{1}-\phi_{1} P_{2}\right)}, \\
\tilde{t}_{2} \geq \frac{\phi_{2}}{\rho \lambda_{2} P_{2}}
\end{gathered}
$$

and

$$
\theta_{2} \geq e^{-\tilde{t}_{1}}-\frac{P_{2}}{P_{2}+P_{1} \omega \phi_{2}} e^{-\tilde{t}_{2}} .
$$

Finally, we tackle the product of fraction and exponent in (A.9) by introducing $\bar{t}_{2}$ as the lower bound of $e^{-\tilde{t}_{2}}$ and $t_{2}$ as the lower bound of $\frac{P_{2}}{P_{2}+P_{1} \omega \phi_{2}} \bar{t}_{2}$. The proof of equivalence between (P8) and (P9) is completed.

\section{REFERENCES}

[1] Y. Cai, Z. Qin, F. Cui, G. Y. Li, and J. A. McCann, "Modulation and multiple access for 5G networks," IEEE Commun. Surv. Tut., vol. 20, no. 1, pp. 629-646, first quarter, 2018.

[2] Y. Liu, Z. Qin, M. Elkashlan, Z. Ding, A. Nallanathan, and L. Hanzo, "Nonorthogonal multiple access for 5G and beyond," Proc. IEEE, vol. 105, no. 12, pp. 2347-2381, Dec. 2017

[3] L. Dai, B. Wang, Y. Yuan, S. Han, C. L. I, and Z. Wang, "Non-orthogonal multiple access for 5G: solutions, challenges, opportunities, and future research trends," IEEE Commun. Mag., vol. 53, no. 9, pp. 74-81, Sep. 2015.

[4] Z. Ding, Y. Liu, J. Choi, Q. Sun, M. Elkashlan, C. L. I, and H. V. Poor, "Application of non-orthogonal multiple access in LTE and 5G networks," IEEE Commun. Mag., vol. 55, no. 2, pp. 185-191, Feb. 2017.

[5] S. M. R. Islam, N. Avazov, O. A. Dobre, and K.-S. Kwak, "Power-domain non-orthogonal multiple access (NOMA) in 5G systems: potentials and challenges," IEEE Commun. Surv. Tut., vol. 19, no. 2, pp. 721-742, second quarter, 2017.

[6] S. M. R. Islam, M. Zeng, O. A. Dobre, and K. Kwak, "Resource allocation for downlink NOMA systems: key techniques and open issues," IEEE Wireless Commun., vol. 25, no. 2, pp. 40-47, Apr. 2018.

[7] S. M. R. Islam, M. Zeng, and O. A. Dobre, "NOMA in 5G systems: exciting possibilities for enhancing spectral efficiency," IEEE $5 G$ Tech Focus, vol. 1, no. 2, Jun. 2017.

[8] Y. Saito, Y. Kishiyama, A. Benjebbour, T. Nakamura, A. Li, and K. Higuchi, "Non-orthogonal multiple access (NOMA) for cellular future radio access," in Proc. IEEE Veh. Technol. Conf. (VTC Spring), Dresden, Germany, Jun. 2013, pp.1-5.

[9] Z. Ding, Z. Yang, P. Fan, and H. V. Poor, "On the performance of nonorthogonal multiple access in 5G systems with randomly deployed users," IEEE Signal Process. Lett., vol. 21, no. 12, pp. 1501-1505, Dec. 2014.

[10] Z. Ding, R. Schober, and H. V. Poor, "A general MIMO framework for NOMA downlink and uplink transmission based on signal alignment," IEEE Trans. Wireless Commun., vol. 15, no. 6, pp. 4438-4454, Jun. 2016.

[11] Y. Tian, A. Nix, and M. Beach, "On the performance of opportunistic NOMA in downlink CoMP networks," IEEE Commun. Lett., vol. 20, no. 5, pp. 998-1001, May 2016.

[12] Y. Liu, Z. Qin, M. Elkashlan, A. Nallanathan, and J. A. McCann, "Nonorthogonal multiple access in large-scale heterogeneous networks," IEEE J. Sel. Areas Commun., vol. 35, no. 12, pp. 2667-2680, Dec. 2017.

[13] Y. Liu, Z. Qin, M. Elkashlan, Y. Gao, and L. Hanzo, "Enhancing the physical layer security of non-orthogonal multiple access in large-scale networks," IEEE Trans. Wireless Commun., vol. 16, no. 3, pp. 1656-1672, Mar. 2017

[14] N. Zhao, W. Wang, J. Wang, Y. Chen, Y. Lin, Z. Ding, and N. C. Beaulieu. "Joint Beamforming and Jamming Optimization for Secure Transmission in MISO-NOMA Networks," IEEE Trans. Commun., to appear.

[15] M. F. Hanif, Z. Ding, T. Ratnarajah, and G. K. Karagiannidis, "A minorization-maximization method for optimizing sum rate in the downlink of non-orthogonal multiple access systems," IEEE Trans. Signal Process., vol. 64, no. 1, pp. 76-88, Jan. 2016.
[16] Y. Liu, M. Elkashlan, Z. Ding, and G. K. Karagiannidis, "Fairness of user clustering in MIMO non-orthogonal multiple access systems," IEEE Commun. Lett., vol. 20, no. 7, pp. 1465-1468, Jul. 2016.

[17] M. Zeng, A. Yadav, O. A. Dobre, G. Tsiropoulos, and V. Poor, "Capacity comparison between MIMO-NOMA and MIMO-OMA with multiple users in a cluster," IEEE J. Sel. Areas Commun., vol. 35, no. 10, pp. 2413-2424, Oct. 2017.

[18] L. Lei, D. Yuan, C. K. Ho, and S. Sun, "Power and channel allocation for non-orthogonal multiple access in 5G systems: Tractability and computation," IEEE Trans. Wireless Commun., vol. 15, no. 12, pp. 85808594, Dec. 2016.

[19] B. Di, L. Song, and Y. Li, "Sub-channel assignment, power allocation, and user scheduling for non-orthogonal multiple access networks," IEEE Trans. Wireless Commun., vol. 15, no. 11, pp. 7686-7698, Nov. 2016.

[20] Z. Qin, X. Yue, Y. Liu, Z. Ding, and A. Nallanathan, "User Association and Resource Allocation in Unified NOMA Enabled Heterogeneous Ultra Dense Networks," IEEE Communications Magazine, vol. 56, no. 6, pp. 86-92, Jun. 2018.

[21] F. Fang, H. Zhang, J. Cheng, and V. C. M. Leung, "Energy-efficient resource allocation for downlink non-orthogonal multiple access network," IEEE Trans. Commun., vol. 64, no. 9, pp. 3722-3732, Sep. 2016.

[22] J. Cui, Y. Liu, Z. Ding, P. Fan, and A. Nallanathan, "Optimal user scheduling and power allocation for millimeter wave NOMA systems," IEEE Trans. Wireless Commun., vol. 17, no. 3, pp. 1502-1517, Mar. 2018.

[23] D. Love, R. Heath, V. Lau, D. Gesbert, B. Rao, and M. Andrews, "An overview of limited feedback in wireless communication systems," IEEE J. Sel. Areas Commun., vol. 26, no. 8, pp. 1341-1365, Oct. 2008.

[24] S. Timotheou and I. Krikidis, "Fairness for non-orthogonal multiple access in 5G systems," IEEE Signal Process. Lett., vol. 22, no. 10, pp. 1647-1651, Oct. 2015.

[25] J. Cui, Z. Ding, and P. Fan, "A novel power allocation scheme under outage constraints in NOMA systems," IEEE Signal Process. Lett., vol. 23, no. 9, pp. 1226-1230, Sep. 2016.

[26] X. Yue, Z. Qin, Y. Liu, Z. Ding, S. Kang, and Y. Chen, "A unified framework for non-orthogonal multiple access," IEEE Trans. Commun., vol. 66, no. 11, pp. 5349-5359, Nov. 2018.

[27] S. Li, M. Derakhshani, and S. Lambotharan, "Outage-constrained robust power allocation for downlink MC-NOMA with imperfect SIC," in Proc. IEEE Int. Conf. Commun. (ICC), Kansas City, MO, USA, May 2018, pp. 1-7.

[28] D. Tweed, M. Derakhshani, S. Parsaeefard, and T. Le-Ngoc, "Outageconstrained resource allocation in uplink NOMA for critical applications," IEEE Access, vol. 5, no. 9, pp. 27636-27648, Nov. 2017.

[29] M. F. Kader, M. B. Shahab, and S. Y. Shin, "Exploiting non-orthogonal multiple access in cooperative relay sharing," IEEE Commun. Lett., vol. 21, no. 5, pp. 1159-1162, Jan. 2017

[30] X. Yue, Y. Liu, S. Kang, A. Nallanathan, and Y. Chen, "Outage performance of two-way relay non-orthogonal multiple access systems," in Proc. IEEE Int. Conf. Commun. (ICC), Kansas City, MO, USA, May 2018, pp. 1-6.

[31] S. Bayat, Y. Li, L. Song, and Z. Han, "Matching theory: Applications in wireless communications," IEEE Signal Process. Mag., vol. 33, no. 6, pp. 103-122, Nov. 2016.

[32] V. Balakrishnan, S. Boyd, and S. Balemi, "Branch and bound algorithm for computing the minimum stability degree of parameter-dependent linear systems," Int. J. Robust Nonlinear Control, vol. 1, no. 4, pp. 295 317, 1991.

[33] A. L. Yuille and A. Rangarajan, "The concave-convex procedure," Neural Comput., vol. 15, no. 4, pp. 915-936, 2003.

[34] Z. Qin and J. A. McCann, "Resource efficiency in low-power wide-area networks for IoT applications," in Proc. IEEE Global Commun. Conf. (GLOBECOM), Singapore, Dec. 2017, pp. 1-7.

[35] E. Bodine-Baron, C. Lee, A. Chong, B. Hassibi, and A. Wierman, "Peer effects and stability in matching markets," in Proc. Symp. Algorithmic Game Theory (SAGT11), Amalfi, Italy: Springer, Oct. 2011, pp. 117-129.

[36] P. C. Weeraddana, M. Codreanu, M. Latva-Aho, and A. Ephremides, "Weighted sum-rate maximization for a set of interfering links via branch and bound," IEEE Trans. Signal Process., vol. 59, no. 8, pp. 3977-3996, Aug. 2011.

[37] CVX Research, Inc. CVX: Matlab software for disciplined convex programming, version 2.0 beta. [Online]. Available: http://cvxr.com/cvx 